ISSN: 2600-5859

\title{
Determinación y georreferenciación de la estructura y composición florística del ecosistema herbazal páramo en la reserva de producción de fauna Chimborazo
}

Determination and georeferencing of the structure and the floristic composition of the herbazal paramo ecosystem in the Chimborazo wildlife production reserve

Robinson Fabricio Peña Murillo. ${ }^{1}$, Vicente Javier Parra León. ${ }^{2}$, Juan Enrique Ureña Moreno . ${ }^{3} \&$ Bryan Andrés Hugo Tubon. ${ }^{4}$

\begin{abstract}
.
DOI: https://doi.org/10.33262/concienciadigital.v3i1.1.1138

Protected areas are the cornerstone of virtually all national and international conservation strategies, as they are a clearly defined geographical space dedicated to the preservation of nature, its ecosystem services and its associated cultural values. In Ecuador it corresponds to $20 \%$ of the total area, and in our province we find the Chimborazo Fauna Production Reserve created on October 26, 1987 with an extension of 58,560 in order to maintain the moor ecosystem's aptitudes. The objective of the research was to determine the structure and floristic composition of the herbaceous páramo ecosystem in the Chimborazo Faunal Production Reserve, taking the Pulinguí community as a study area. For the sampling the Gloria methodology was specified, installing 13 plots of $5 \times 5 \mathrm{~m}$, in which the species were recorded from codes and comparisons with secondary information. 780 individuals were registered in 17 orders and 24 families, the most numerous being polar and asteral, with 167 and 166 individuals respectively, the data confirmed the high floristic diversity and ecological importance of these ecosystems, so it is recommended to keep an updated inventory and strategies that specify its conservation.
\end{abstract}

Keywords: Biodiversity Conservation, Diversity, Protected natural area, Species Resumen.

\footnotetext{
${ }^{1}$ Escuela Superior Politécnica de Chimborazo, Riobamba Ecuador, robinson.pena@espoch.edu.ec

${ }^{2}$ Escuela Superior Politécnica de Chimborazo, Riobamba Ecuador, vicente.parra@espoch.edu.ec

${ }^{3}$ Escuela Superior Politécnica de Chimborazo, Riobamba Ecuador, juan.urena@espoch.edu.ec

${ }^{4}$ TONETO CIA. LTDA., Riobamba Ecuador, bryanandres1501204596@ hotmail.com
} 
Las áreas naturales protegidas son un recurso de vital importancia en las estrategias nacionales e internacionales de conservación, pues son un espacio geográfico muy bien definido y dedicado a la preservación de los recursos naturales, los servicios ecosistémicos inherentes y los valores culturales. En el Ecuador continental e insular corresponden al 20\% de la superficie total, y en nuestra provincia encontramos la Reserva de Producción de Fauna Chimborazo creada el 26 de octubre de 1987 con una extensión de 58.560 con el propósito de mantener las aptitudes del ecosistema de páramo. El objetivo de la investigación fue determinar la estructura y composición florística del ecosistema herbazal de páramo en la Reserva de Producción Faunística Chimborazo, tomando como zona de estudio la comunidad Pulinguí. Para el muestreo se utilizó la metodología Gloria, instalándose 13 parcelas de $5 \times 5 \mathrm{~m}$, en las cuales fueron registradas las especies a partir de códigos y comparadas con información secundaria. Se registraron 780 individuos en 17 órdenes y 24 familias, siendo las más numerosos las poales y asterales, con 167 y 166 individuos respectivamente, éstos datos confirman la alta diversidad florística e importancia ecológica de éste ecosistema por lo que, se recomienda mantener un inventario actualizado y estrategias que permitan su conservación.

Palabras claves: Conservación, Diversidad, Áreas naturales protegidas, Especies

\section{Introducción.}

La áreas naturales declaradas como protegidas son un espacio geográfico muy bien definido dedicadas a la gestión y conservación de los recursos naturales, mediante medios legales u otros tipos de medios eficaces para conseguir la conservación a largo plazo de la naturaleza y de sus servicios ecosistémicos y sus valores culturales asociados (Dudley, 2008).

Estos espacios siguen siendo de vital importancia para las estrategias nacionales e internacionales que buscan la conservación de las especies en todos los niveles, cabe reslatar que, estas estrategias cuentan con el apoyo de gobiernos e instituciones locales, nacionales e internacionales. Dado que las áreas protegidas constituyen el principal instrumento de conservación que salvaguarda en la actualidad el 14\% de la superficie terrestre (incluida la Antártida) y el 3,41\% de la superficie marina del planeta, a través de 209.428 áreas, en $32^{\prime} 868.673 \mathrm{~km}$ (Deguignet et al., 2014), en muchos casos han sido determinantes para proteger ciertas áreas donde se desarrollan comunidades humanas con un alto valor cultural (Dudley, 2008).

En la actualidad, en el Ecuador encontramos 56 reservas naturales, correspondientes al $20 \%$ de la superficie total del país, distribuidas en las regiones continentales y en las Islas Galápagos (Ministerio del Ambiente, 2017). Como parte del Sistema Nacional de Áreas Protegidas (SNAP)

y con una importancia tanto en el tema de la conservación de las especies, para el sector turístico y cultura, encontramos la Reserva de Producción de Fauna Chimborazo (RPFCH) (Ministerio del ambiente , 2014). La Reserva de Producción de Fauna Chimborazo, fue creada con fines de 
conservación de camélidos andinos, el 26 de octubre de 1987, y se localiza en las provincias de Chimborazo, Bolívar y Tungurahua, con una extensión aproximada de de 58.560 ha, y con una altura que varía desde los 3.800 a 6.248 m.s.n.m., presenta un clima frio andino con temperaturas desde el $0^{\circ}$ a $10^{\circ} \mathrm{C}$. Su propósito es mantener las funciones claves del ecosistema basados en parámetros ecológicos, además se ha planteado como objetivo principal de ésta reserva, el conservar el hábitat de los camélidos andinos.

En el mismo sentido, se pretende establecer infraestructura, servicios y brindar las facilidades para la investigación del ecosistema páramo, desarrollando de ésta manera actividades que fomenten la recreación y el turismo sustentable (MAE , 2017). Dentro de las cuatro zonas de vida que se puede concentrar en la Reserva de Producción Faunística Chimborazo, están siete tipos de ecosistema: Herbazal y Arbustal siempreverde subnival de Páramo, Herbazal de Páramo, Arbustal siempre verde y Herbazal de Páramo, Herbazal inundable del Páramo, Herbazal ultra húmedo subnival de Páramo, Herbazal húmedo montano alto superior de Páramo (Ministerio del ambiente , 2014).

El páramo es un ecosistema natural sobre el límite de bosque cerrado en los Andes del Norte, dominado por pajonales, rosetales, arbustales, humedales y pequeños bosquetes. Es un ecosistema de clima frío y es muy frágil a los cambios en el uso de la tierra, por lo que su potencial para el uso productivo es, en términos generales, muy limitado (Mena \& Hofstede, 2006). En el Ecuador, el páramo cubre alrededor de 1.260 .000 ha, es decir, éste ecosistema cubre aproximadamente un $6 \%$ del territorio nacional continental (Mena P. , 2010).

La provincia de Chimborazo, con una extensión de 648.124 ha, posee más de 236.000 ha de ecosistema de páramo, es decir, estaríamos hablando del $36,9 \%$ de la superficie de la provincia y otras 83.800 ha son bosque andino y alto andino, es decir, representa el 13,1\% de la superficie de la provincia (Beltrán, Bustamante, \& Arguello, 2014). A nivel nacional, esto representa el 17,7\% del total de superficie de páramo en el país (Beltrán, y otros, 2009), albergando una gran diversidad florística en ellos. En este sentido la importancia de la reserva radica en los diferentes ecosistemas propios de los andes que alberga el área, distribuidos en diferentes pisos altitudinales y en algunos casos colindando con asentamientos humanos, tal es el caso de la comunidad Punlinguí, misma que presenta un alto grado de biodiversidad en su ecosistema herbazal de páramo.

El ecosistema herbazal de páramo al ser uno de los más extensos de la Reserva de Producción de Fauna Chimborazo, posee abundante riqueza florística, existen especies de plantas que no crecen en ningún otro bioma (endemismo), debido a su morfología actúan como esponjas, ayudan en la regulación hídrica y debido a los factores climáticos la descomposición es lenta ayudando a almacenar carbono en el suelo, de ahí su valiosa importancia ecológica para la flora y fauna del sector y de las poblaciones que utilizan los recursos hídricos para su subsistencia, pero a su vez, posee una fragilidad evidente, puesto que la vegetación apenas tolera bajas frecuencias de quema 
ISSN: 2600-5859

y pastoreo (MAE, 2015). La agricultura, el sobrepastoreo, la quema de pajonal, deforestación y la canalización de agua son actividades cotidianas de la comunidad Pulinguí y de las demás comunidades presentes en la reserva causando efectos negativos en la composición y estructura de sus suelos, llevando a la alteración y destrucción de la cobertura vegetal y aumentando así el riesgo de un colapso inminente.

La estructura y composición florística es un estudio permite comprender el estado ecológico y necesidades de manejo de un bosque para promover procesos y funciones naturales del ecosistema y mantener la diversidad, por tales motivos las características florísticas y estructurales revisten una importancia en la planificación del manejo y conservación de los recursos forestales (Méndez \& Sáenz, 1986). A pesar de ser privilegiados con una gran biodiversidad florística presentes en éstos ecosistemas, las poblaciones se encuentran en conflictos constantemente debido a las actividades propias como: la quema de pajonales, el sobrepastoreo, la canalización de agua, son actividades que de alguna manera están destruyendo la cobertura vegetal y por ende alterando los servicios ecosistémicos que el páramo provee.

Con esta premisa, se debe entender que el conocimiento de la composición y biodiversidad florística de un ecosistema es importante porque nos permite planificar procesos de conservación y gestión sustentable de los servicios ecosistémicos y culturales asociados.

En ese sentido, el objetivo de la investigación es determinar la estructura y composición florística del ecosistema herbazal de páramo en la Reserva de Producción Faunística Chimborazo, tomando como zona de estudio la comunidad Pulinguí-Chimborazo, ubicada en la Reserva. La información generada permitirá a los administradores de la Reserva orientar una gestión efectiva que priorice la conservación de los ecosistemas fragmentados, además de concienciar a los pobladores de la zona sobre el valor de mantener este ecosistema y establecer compromisos de conservación con las comunidades.

\section{Metodología}

Para determinar la composición y estructura florística del ecosistema herbazal de páramo de la comunidad Pulinguí, ubicada en la Reserva de producción faunística Chimborazo se realizó el siguiente procedimiento:

\section{Ubicación del área de estudio:}

Para la investigación se ha considerado representativamente la comunidad Pulinguí, parroquia San Juan, cantón Riobamba, provincia de Chimborazo, y que se inscribe dentro de la jurisdicción de la Reserva de Producción de Fauna Chimborazo. En la figura 1 se puede apreciar el área de estudio. 


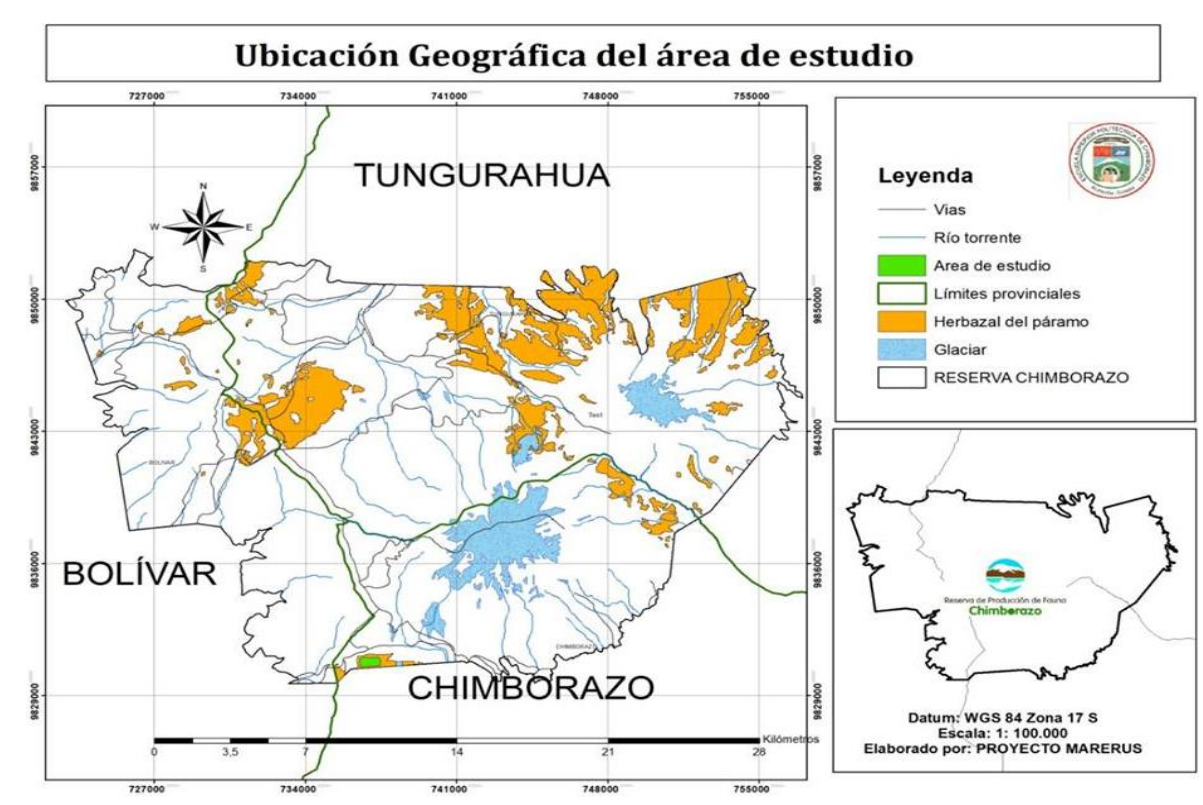

Figura 1. Ubicación geográfica del área de estudio Elaborado por: Proyecto MARERUS

\section{Límites}

Para el establecimiento de los límites del estudio, se consideraron las fronteras geográficas de la comunidad Pulinguí, limitando al Norte con la Parroquia San Andrés, al Sur con la comunidad Cimborazo, al Este con la comunidad Chorrera Mirador y al Oeste con la comunidad Ganguis, datos que se aprecian en la Tabla 1.

\begin{tabular}{ll}
\hline Norte & Parroquia San Andrés \\
\hline Sur & Comunidad Chimborazo \\
Este & Comunidad Chorrera Mirador \\
Oeste & Comunidad Ganquis (Provincia de Bolívar) \\
\hline
\end{tabular}

Tabla1. Límites del área de estudio

Elaborado por: Investigación de campo, 2018

\section{Adaptación de la metodología GLORIA}

Para realizar el muestreo se utilizó la adaptación de la metodología Gloria, para la cual se realizaron dos salidas de campo, una para delimitar el área de estudio utilizando el sistema de información geográfica con un levantamiento topográfico con GNSS (método RTK), además se realizó la georreferenciación del área apoyados en el software ArcGIS 10.3, dejando para la segunda salida de campo el muestreo sistemático. 
En el área de la investigación, se instalaron 13 parcelas de $5 \times 5 \mathrm{~m}$. Luego, en cada parcela se realizó la subdivisión en cuadrantes de 1 x $1 \mathrm{~m}$., las observaciones de vegetación se llevaron a cabo únicamente en los cuatro cuadrantes ubicados en las esquinas o extremos, para evitar las cuadrículas internas que podrían estar alterados por el pisoteo del investigador, con esto evitamos alteraciones en la composición florística a lo largo del muestreo, como se puede apreciar en la figura 2. Cada uno de los cuadrantes de 1x1 m. se subdividieron en cuadriculas de 0.1 x $0.1 \mathrm{~m}$, para ello se utilizó un armazón de madera con un enrejado formado por hilos finos que delimite 100 celdilla de 0.1 x $0.1 \mathrm{~m}$., de acuerdo a la metodología del manual GLORIA adaptado por (Rodríguez, 2011) y (Caranqui, Haro, Salas, \& Palacios, 2013). $\quad$ En la Figura 2 se pueden apreciar el mapa por parcela de muestreo.

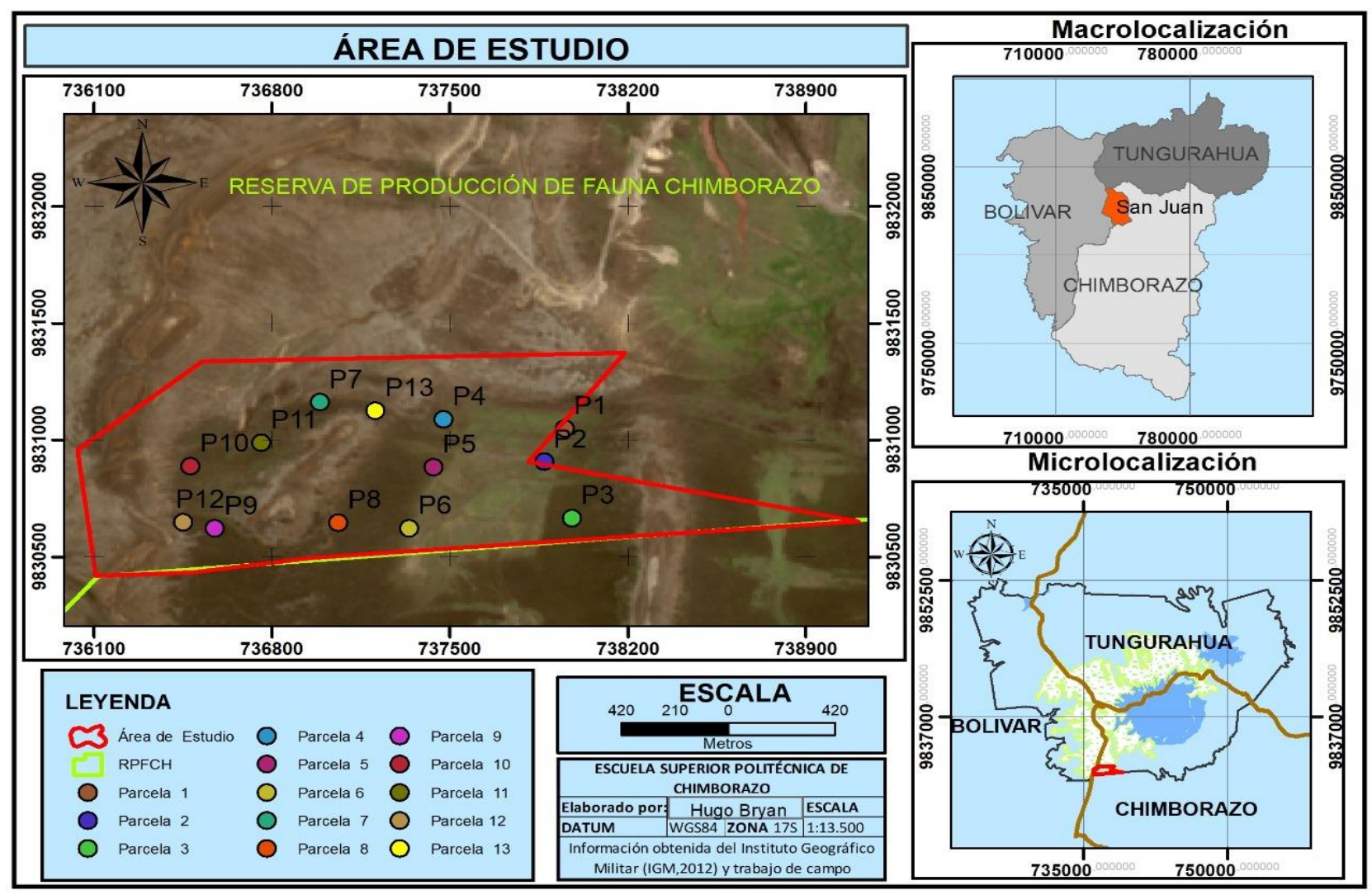

Figura 2. Mapa por parcelas de muestreo

Elaborado por: Investigación de campo, 2018

\section{Registro de especies}

El registro de las especies de realizó en base a códigos asignados con caracteres y números según las parcelas y cuadrantes establecidos en la zona de estudio, como se aprecia en la Tabla 2. 
ISSN: 2600-5859

Vol. 3, $\mathrm{N}^{\circ} 1.1$, p. 168-180, febrero, 2020

\begin{tabular}{ccccccccc}
\hline & \multicolumn{10}{c}{ Parcela } \\
\hline Especie & A & B & C & D & E & F & G & H \\
1 & A01 & B01 & C01 & D01 & E01 & F01 & G01 & H01 \\
2 & A02 & B02 & C02 & D02 & E02 & F02 & G02 & H02 \\
3 & A03 & B03 & C03 & D03 & E03 & F03 & G03 & H03 \\
4 & A04 & B04 & C04 & D04 & E04 & F04 & G04 & H04 \\
5 & A05 & B05 & C05 & D05 & E05 & F05 & G05 & H05 \\
6 & A06 & B06 & C06 & D06 & E06 & F06 & G06 & H06 \\
7 & A07 & B07 & C07 & D07 & E07 & F07 & G07 & H07 \\
\hline
\end{tabular}

Tabla 2. Registro por Códigos

Fuente: Equipo de investigación, 2018

\section{Identificación y comparación de especies}

Para la identificación de las especies fotografiadas se realizó búsquedas en sitios web como: Trópicos, Herbario JBB en Línea, biovirtual.unal.edu.co, jardín.lautaret, herbarivirtual.uib.es, Catálogo virtual de Flora de alta Montaña, libro "Flora del Páramo del Cajas" y libro "Plantas Vasculares de los Bosques de Polylepis en los Páramos de Oyacachi”, con la finalidad de determinar orden, familia y nombres científicos de las especies vegetales.

\section{Resultados}

Una vez identificada el área de estudio, se realizó el muestro según el procedimiento respectivo (Adaptación de la metodología Gloria), obteniéndose los resultados de la caracterización de la flora de la RPFCH que se pueden apreciar en la Figura 4. Así, se encontraron 780 individuos, repartidos en 17 órdenes y 26 familias en las 13 parcelas. El orden con más individuos encontrados es el poales con 167 individuos seguido del orden asterales con 166 y el orden con un menor número de individuos es el myrtales con 2 seguido del orden malphigiales con 6 individuos. Esta distribución, coincide con lo afirmado por el (Ministerio de Ambiente del Ecuador, 2012) que, en sus investigaciones, inventarios y mapas de distribución de la vegetación, ha encontrado el orden poales como el más numeroso del ecosistema herbazal páramo.

Se determinó de ésta manera una alta biodiversidad en las zonas de muestreo, cabe resaltar que la investigación fue realizada en zonas que no han sufrido de intervención antropogénica, por lo que muestran diversidad y composición florística típica de estos ecosistemas como lo afirma Mena (2016) , quien determina que los páramos pese a ubicarse en altitudes superiores a los 3000 m.s.n $\mathrm{m}$, presenta alta biodiversidad si no han sido intervenido por los seres humanos, siendo así, los páramos son considerados los ecosistemas de montaña más diversos del planeta.

En lo que se refiere al orden asterales, en la investigación fueron encontrados 166 individuos, 
siendo el segundo en cantidad después del orden poales. Este dato coincide por lo afirmado por Mena (2006) y por Beltran (2009) quienes aseguran que el orden asterales es el más numeroso de todos, con cerca de 28000 especies y con una distribución bastante cosmopolita y con un sinnúmero de usos.

En el mismo sentido, Mena (2006) y Hofstede (2014), afirman que la distribución del orden myrtales corresponde a ecosistemas tropicales y subtropicales, por lo que, coincide en la caracterización de la presente investigación en la cual fueron encontrados dos individuos solamente para el orden myrtales, por estar ubicada el área de investigación en un ecosistema a más de 3200 m.s.n.m. La distribución del orden malphigiales según los mismos autores también se encuentra ampliamente en los trópicos, por lo que, coincide con la presente investigación en la cual fueron encontrados 6 individuos para éste orden.

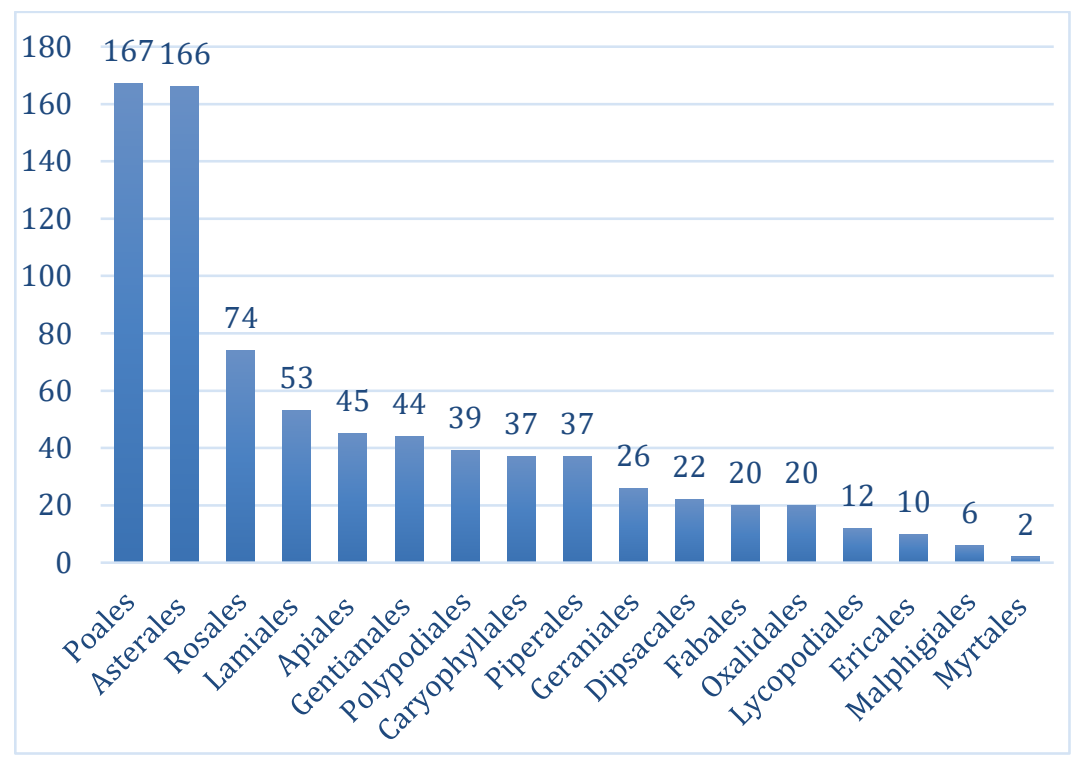

Figura 3. Número de individuos por orden

Elaborado por: Investigación de campo, 2018

En lo que se refiere a la distribución de individuos por especies, de la totalidad de especies encontradas, la más numerosa fue Calamagrostis intermedia (J. Presl) Steud del orden de las Poales con 154 individuos, encontrados en las 13 parcelas como se puede apreciar en la Tabla 3. Este dato coincide con lo encontrado en Mena (2006) quien afirma que Calamagrostis intermedia (J. Presl) Steud es la especie más común y numerosa de los páramos ecuatorianos, siendo además una especie nativa de éste ecosistema.

La especie en segundo lugar (según la distribución en las parcelas de muestreo) fue la Lachemilla orbiculata (Ruiz \& Pav.) Rydb, perteneciente al orden de las Rosales, seguidas de Werneria 
ISSN: 2600-5859

pygmaea Gillies ex Hook. \& Arn y Clinopodium nubigenum (Kunth) con 46 y 42 especies. Esta distribución está en concordancia con lo afirmado por (Ministerio de Ambiente del Ecuador, 2012) y Mena (2006) quienes han identificado a éste tipo de plantas vasculares como las principales del ecosistema Herbazal de Páramo, por ser pioneras y de amplia cobertura en los páramos, ésta distribución lo podemos observar en la distribución por parcelas e individuos de la Tabla 3.

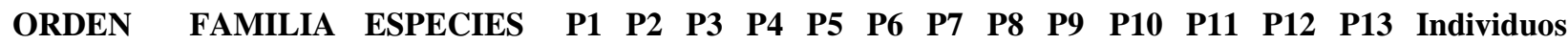

\begin{tabular}{|c|c|c|c|c|c|c|c|c|c|c|c|c|c|c|c|c|c|}
\hline 1 & Poales & Poaceae & $\begin{array}{l}\text { Calamagrost } \\
\text { is intermedia } \\
\text { (J. Presl) } \\
\text { Steud }\end{array}$ & 12 & 17 & 10 & 17 & 14 & 14 & 11 & 14 & 11 & 7 & 11 & 6 & 10 & 154 \\
\hline 2 & Rosales & Rosaceae & $\begin{array}{l}\text { Lachemilla } \\
\text { orbiculata } \\
\text { (Ruiz \& } \\
\text { Pav.) Rydb. }\end{array}$ & 16 & 8 & 8 & 9 & 5 & 1 & 2 & 3 & 0 & 2 & 0 & 2 & 8 & 64 \\
\hline 3 & Asterales & Asteraceae & $\begin{array}{l}\text { Werneria } \\
\text { pygmaea } \mathrm{Gi} \\
\text { llies ex } \\
\text { Hook. \& Arn }\end{array}$ & 10 & 18 & 0 & 0 & 0 & 2 & 0 & 1 & 7 & 0 & 0 & 0 & 8 & 46 \\
\hline 4 & Lamiales & Lamiaceae & & 0 & 0 & 0 & 3 & 0 & 26 & 0 & 0 & 4 & 9 & 0 & 0 & 0 & 42 \\
\hline
\end{tabular}

Tabla 3. Distribución de individuos los más numerosos por parcelas y por especies

Fuente: Investigación de campo (2018)

En la Tabla 4 podemos observar la distribución de los individuos menos numerosos por especies, es así que fueron identificados solamente un individuo de Carex pichinchensis Kunth, que según Mena (2006) esta especie es propia de zonas intervenidas, por lo que coincide con los datos de la investigación, ya que los muestreos realizados fueron en espacios naturales donde no han sufrido intervención antropogénica.

En lo que se refiere a Puya clava herculis Mez \& Sodiro, se encontró un solo individuo, coincide con lo asegurado por Mena (2006) y (Ministerio de Ambiente del Ecuador, 2012), ya que esta especie se encuentra en el libro rojo de las especies endémicas en peligro de extinción. Su escaso número de individuos se debe a que al tratarse de una especie monocárpica es susceptible al fuego ocasionado por la quema de pajonales muy comunes en la zona andina. 


\begin{tabular}{lllc}
\hline \multicolumn{1}{c}{ ORDEN } & FAMILIA & \multicolumn{1}{c}{ ESPECIE } & Individuos \\
\hline Myrtales & Melastomataceae & $\begin{array}{l}\text { Miconia salicifolia } \text { (Bonpl. ex } \\
\text { Naudin) Naudin }\end{array}$ & 2 \\
Asterales & Asteraceae & $\begin{array}{l}\text { Monticalia arbutifolia (Kunth) } \\
\text { C. Jeffrey }\end{array}$ & 2 \\
Poales & Bromeliaceae & $\begin{array}{l}\text { Puya clava herculis Mez \& } \\
\text { Sodiro }\end{array}$ & 1 \\
Poales & Cyperaceae & Carex pichinchensis Kunth & 1 \\
\hline
\end{tabular}

Tabla 4. Distribución de individuos por especie (los menos numerosos).

Fuente: Investigación de campo, 2018

En el mismo contexto, en la figura 4 se observa la acumulación de especies por parcela, es decir la cantidad de muestreo realizados hasta que el número de especies se estabilice. Así, en el eje $\mathrm{X}$ se muestra el esfuerzo de muestreo efectuado y en el eje $\mathrm{Y}$ el número de especies encontradas para cada nivel de muestreo dado. Es decir que se necesitaron instalar 13 parcelas para alcanzar la asíntota, en donde el número de especies se estabiliza e indica que el esfuerzo de muestreo ha sido suficiente para inventariar toda la vegetación herbácea del ecosistema herbazal de páramo de la comunidad Pulinguí., esto según la recomendación de Rodriguez (2011).

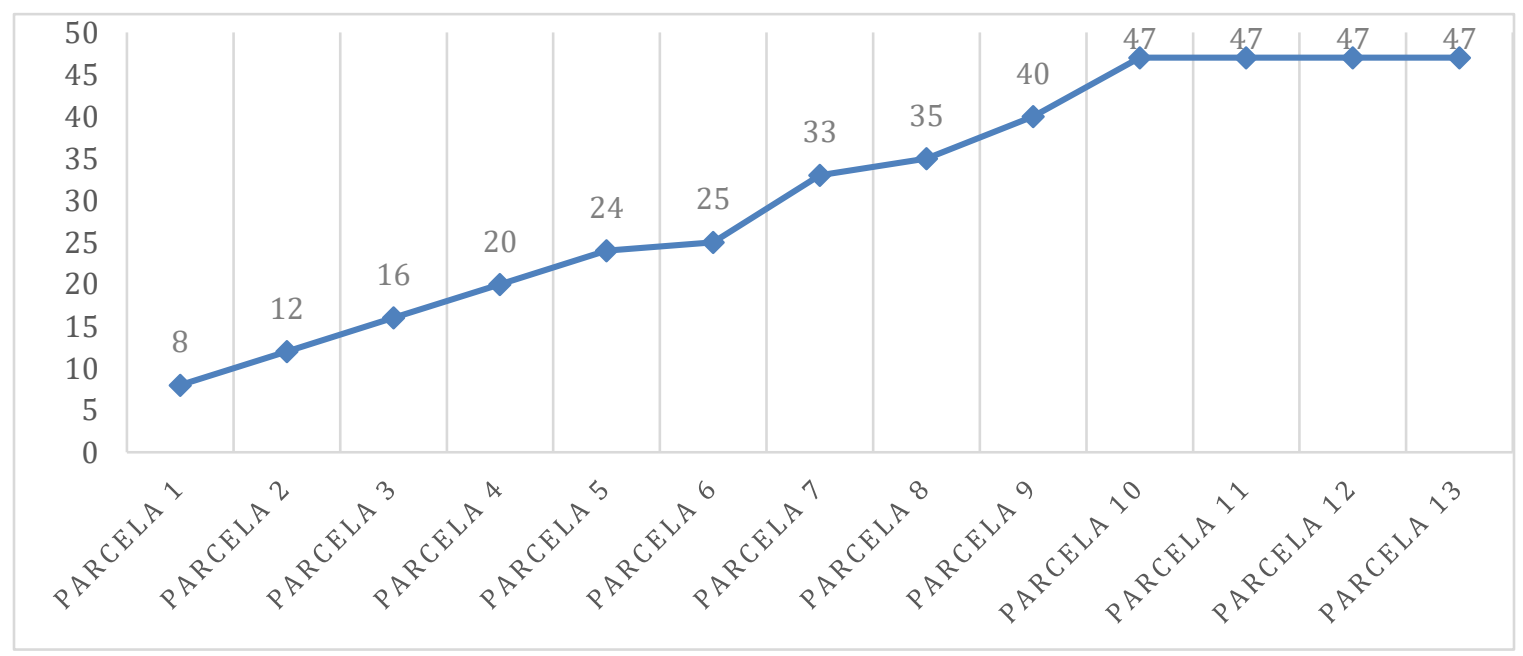

Figura 4. Curva de acumulación de especies por parcela

Elaborado por: Investigación de campo, 2018 
ISSN: 2600-5859

\section{Conclusiones.}

- Después de realizado el inventario de vegetación herbácea de la comunidad Pulinguí en la Reserva de Producción de Fauna Chimborazo, concluye con los siguientes datos cuantitativos: un total de 780 individuos, repartidos en 17 órdenes y 26 familias, confirmándose la alta diversidad presente en éste ecosistema.

- Del total de individuos encontrados, el orden con mayor abundancia fue el de las poales con 167, seguido del orden asterales con 166 individuos. En tanto que, la especie más númerosa fue Calamagrostis intermedia (J. Presl) Steud del orden de las Poales con 154 individuos, encontrados en las 13 parcelas, en segundo lugar se encontró la especies Lachemilla orbiculata (Ruiz \& Pav.) Rydb, perteneciente al orden de las Rosales, seguidas de Werneria pygmaea Gillies ex Hook. \& Arn y Clinopodium nubigenum (Kunth) con 46 y 42 especies respectivamente.

- El ecosistema herbazal de páramo es un ecosistema que alberga una gran cantidad de especies de vegetación en su mayoría tipo herbácea, razón por la cual es recomendable realizar inventarios para llevar registros de las especies presentes en el lugar con la finalidad de mantenerlas bajo monitoreo y hacer comparaciones cada cierto tiempo comprobando de esta manera si las especies registradas se mantienen o disminuyen.

- Para recopilar información se recomienda elaborar una herramienta de campo adecuada, fácil y sencilla que se ajuste a los requerimientos del caso de estudio ya que se debe tomar en cuenta algunas variables como son la disponibilidad de tiempo, tipo de variables a analizar y contexto de la investigación, con estos detalles se asegura la fiabilidad de la información obtenida y la eficiencia en la elaboración de la investigación.

- Fue necesario instalar 13 parcelas para alcanzar la asíntota, es decir el número de especies identificadas se estabilizó en la parcela No. 13, indicando que el esfuerzo de muestreo ha sido suficiente para inventariar toda la vegetación herbácea del ecosistema herbazal de páramo de la comunidad Pulinguí.

\section{Referencias bibliográficas.}

Rodríguez, M. (2011). Diversidad Florística; Páramo de Almohadillas; Yatzaptzán (Comunidad); Ambato (Cantón); Pilahuín (Parroquia); Tungurahua. Obtenido de http://dspace.espoch.edu.ec/handle/123456789/741

Méndez, J., \& Sáenz, L. (1986). Estructura y composición de dos comunidades arbóreas de la parte noreste de la Cordillera de Talamanca, Costa Rica. Obtenido de http://www.scielo.org.mx/scielo.php?script=sci_arttext\&pid=S1405-04712016000300037

Mena, P. (2010). Los páramos ecuatorianos: diversos, frágiles y estratégicos. Obtenido de https://afese.com/img/revistas/revista54/paramos.pdf 
ISSN: 2600-5859

Beltrán, K., Bustamante, F., \& Arguello, M. (2014). Los páramos de Chimborazo. Un estudio socio ambiental para la toma de decisiones. Obtenido de file://C:/Users/USER/Downloads/LospramosdeChimborazo.pdf

Beltrán, K., Salgado, S., Cuesta, F., León, S., Romoleroux, K., Ortíz, E., . . Alexandra, V. (2009). Distribución Espacial, Sistemas Ecológicos y Caracterización Florística de los Páramos en el Ecuador. Obtenido de http://www.flacsoandes.edu.ec/libros/digital/43577.pdf

Caranqui, J., Haro, W., Salas, F., \& Palacios, C. (2013). Diversidad y Similitud de los Páramos De la Provincia de Chimborazo. Obtenido de http://ingenieria.ute.edu.ec/enfoqueute/index.php/revista/article/view/86/91

Deguignet, M., Bignoli, D., Harrison, J., MacSharry, B., Burgess, N., \& Kingston, N. (2014). United Nations Listof Protected Areas. Obtenido de https://wdpa.s3.amazonaws.com/WPC2014/2014_UN_LIST_REPORT_EN.pdf

Dudley, N. (2008). Directrices para la aplicación de las categorías de gestión de áreas protegidas. Obtenido de https://portals.iucn.org/library/efiles/documents/paps-016-es.pdf

Hofstede, R., Calles, J., López, V., Polanco, R., Torres, F., \& Ulloa, J. (2014). Los páramos andinos . Obtenido de Robert Hofstede, Juan Calles, Víctor López, Rocío Polanco, Fidel Torres, Janett Ulloa, Adriana Vásquez: https://portals.iucn.org/library/sites/library/files/documents/2014-025.pdf

Hofstede, R., Calles, J., López, V., Polanco, R., Torres, F., Ulloa, J., \& Vásquez, A. (2014). LOS PÁRAMOS ANDINOS. Obtenido de https://portals.iucn.org/library/sites/library/files/documents/2014-025.pdf

Mena, P., \& Hofstede, $\quad$ R. http://beisa.dk/Publications/BEISA\%20Book\%20pdfer/Capitulo\%2006.pdf. Obtenido de http://beisa.dk/Publications/BEISA\%20Book\%20pdfer/Capitulo\%2006.pdf.

\section{【 Ciencia}


ISSN: 2600-5859

Vol. 3, $\mathrm{N}^{\circ} 1.1$, p. 168-180, febrero, 2020

PARA CITAR EL ARTÍCULO INDEXADO.

Peña Murillo, R. F., Parra León, V. J., Ureña Moreno , J. E., \& Hugo Tubon, B. A. (2020). Determinación y georreferenciación de la estructura y composición florística del ecosistema herbazal páramo en la reserva de producción de fauna Chimborazo . ConcienciaDigital, 3(1.1), 168-180. https://doi.org/10.33262/concienciadigital.v3i1.1.1138

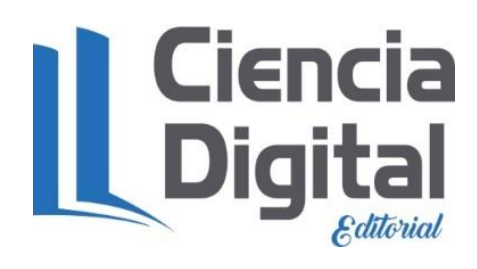

El artículo que se publica es de exclusiva responsabilidad de los autores y no necesariamente reflejan el pensamiento de la Revista Conciencia Digital.

El artículo queda en propiedad de la revista y, por tanto, su publicación parcial y/o total en otro medio tiene que ser autorizado por el director de la Revista Conciencia Digital.
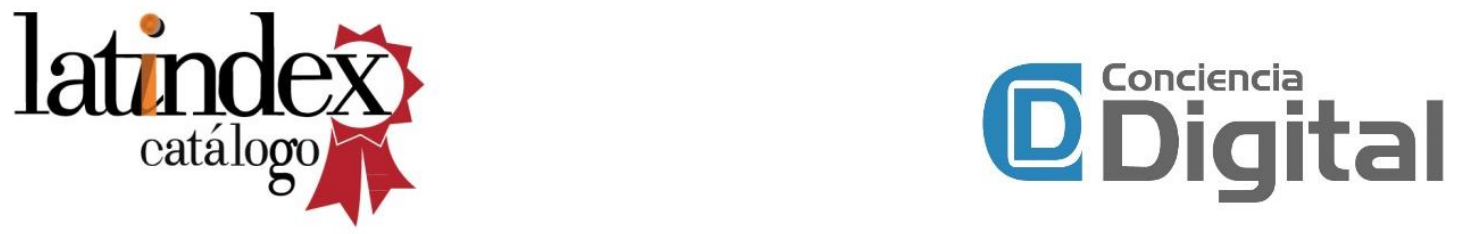